\title{
Mechanical chest compression devices in the helicopter emergency medical service in Switzerland
}

Urs Pietsch ${ }^{1,2^{*}}$, David Reiser ${ }^{3}$, Volker Wenzel ${ }^{4}$, Jürgen Knapp ${ }^{2,3}$, Mario Tissi ${ }^{5}$, Lorenz Theiler ${ }^{3,5}$, Simon Rauch ${ }^{6,7}$, Lorenz Meuli ${ }^{8}$ and Roland Albrecht ${ }^{1,5}$

\begin{abstract}
Background: Over the past years, several emergency medical service providers have introduced mechanical chest compression devices (MCDs) in their protocols for cardiopulmonary resuscitation (CPR). Especially in helicopter emergency medical systems (HEMS), which have limitations regarding loading weight and space and typically operate in rural and remote areas, whether MCDs have benefits for patients is still unknown. The aim of this study was to evaluate the use of MCDs in a large Swiss HEMS system.
\end{abstract}

Materials and methods: We conducted a retrospective observational study of all HEMS missions of Swiss Air rescue Rega between January 2014 and June 2016 with the use of an MCD (Autopulse ${ }^{\oplus}$ ). Details of MCD use and patient outcome are reported from the medical operation journals and the hospitals' discharge letters.

Results: MCDs were used in 626 HEMS missions, and 590 patients (94\%) could be included. 478 (81\%) were primary missions and 112 (19\%) were interhospital transfers. Forty-nine of the patients in primary missions were loaded under ongoing CPR with MCDs. Of the patients loaded after return of spontaneous circulation (ROSC), 20 (7\%) experienced a second CA during the flight. In interhospital transfers, 102 (91\%) only needed standby use of the MCD. Five (5\%) patients were loaded into the helicopter with ongoing CPR. Five (5\%) patients went into CA during flight and the MCD had to be activated. A shockable cardiac arrhythmia was the only factor significantly associated with better survival in resuscitation missions using MCD (OR 0.176, 95\% confidence interval 0.084 to $0.372, p<0.001$ ).

Conclusion: We conclude that equipping HEMS with MCDs may be beneficial, with non-trauma patients potentially benefitting more than trauma patients.

Keywords: AutoPulse, Mechanical chest compression devices, Cardiopulmonary arrest, Cardiopulmonary resuscitation, Helicopter emergency medical services, Load-distributing band CPR device

\footnotetext{
* Correspondence: urs.pietsch@kssg.ch

'Department of Anaesthesiology and Intensive Care Medicine, Cantonal Hospital St. Gallen, Rorschacher Strasse 95, 9007 St. Gallen, Switzerland

${ }^{2}$ Air Zermatt, Emergency Medical Service, Zermatt, Switzerland

Full list of author information is available at the end of the article
}

(c) The Author(s). 2020 Open Access This article is licensed under a Creative Commons Attribution 4.0 International License, which permits use, sharing, adaptation, distribution and reproduction in any medium or format, as long as you give appropriate credit to the original author(s) and the source, provide a link to the Creative Commons licence, and indicate if changes were made. The images or other third party material in this article are included in the article's Creative Commons licence, unless indicated otherwise in a credit line to the material. If material is not included in the article's Creative Commons licence and your intended use is not permitted by statutory regulation or exceeds the permitted use, you will need to obtain permission directly from the copyright holder. To view a copy of this licence, visit http://creativecommons.org/licenses/by/4.0/. The Creative Commons Public Domain Dedication waiver (http://creativecommons.org/publicdomain/zero/1.0/) applies to the data made available in this article, unless otherwise stated in a credit line to the data. 


\section{Introduction}

High-quality chest compressions, minimal hands-off times, and early external defibrillation are crucial for survival with good neurological outcome in patients with cardiac arrest (CA), as underlined by the current resuscitation guidelines [1-3]. However, maintaining highquality cardiopulmonary resuscitation (CPR) is often challenging in the prehospital setting, in particular during evacuation and transport $[4,5]$. Over the past years, a number of institutions providing emergency medical care have introduced mechanical chest compression devices (MCDs) in their CPR protocols [6-8]. AutoPulse (Zoll Medical, Chelmsford, MA, USA) and LUCAS ${ }^{\text {тм }}$ (Physio-Control, Redmond, WA, USA) are the two most frequently used devices worldwide [9].

The Circulation Improving Resuscitation Care (CIRC), LUCAS in cardiac arrest (LINC) and Prehospital Randomized Assessment of a Mechanical Compression Device (PARAMEDIC) trials have assessed the effect of MCDs on survival. In these large prehospital trials, no benefit in patient outcome was shown, hence the routine use of MCDs is not recommended in ERC and AHA CPR guidelines [10-12]. These CPR guidelines, however, suggest the use of MCDs in situations where providing manual chest compressions is impractical, as a bridge to advanced therapies, or when provider safety is compromised. This applies, for example, to helicopter emergency medical services (HEMS). Common European EMS helicopters such as the H145, EC135 or AW109 have limited space and height in the cabin, and providing high-quality chest compressions in a flying helicopter or in a remote environment with limited personnel and space is often impossible.

In Switzerland, four organizations provide physicianstaffed HEMS operations 24/7, including primary (prehospital retrieval) and secondary (inter-hospital transfer) missions around the clock.

The less space available for the HEMS crew and patient during the transport, the greater the advantages of MCDs. Many medical professionals therefore support equipping HEMS with MCDs. However, this could result in frequent transport of patients in persistent CA with dismal prognoses, thereby not only producing direct costs for transport and treatment but also harming other patients indirectly by blocking valuable emergency medical care resources [13].

In this retrospective study, we sought to investigate frequency and circumstances surrounding use of an MCD in a large Swiss helicopter emergency medical service (HEMS), and to analyze use in terms of compliance with CPR guidelines and patient outcome.

\section{Methods}

\section{Design}

We conducted a retrospective study examining the period lasting from December 31, 2013, to June 30, 2016
(912 days). The institutional review board of the Bern cantonal ethics committee (KEK) reviewed the study design and granted permission for use of patient data without individual consent, according to the federal act on research involving human beings, and the ordinance on human research with the exception of clinical trials. The permission covered the use of patient data collected during the HEMS operation and related hospital stays (KEK Bern, September 13, 2016, reference number 2016-01473).

\section{Setting and population}

Swiss Air-Rescue (Rega) conducts around 11,000 HEMS missions annually. In 2013 Rega operated 13 helicopter bases, which are distributed throughout the country in such a way that they can fly to any location at any time within its operational area within $15 \mathrm{~min}$. The helicopter fleet comprises 7 Eurocopter EC145 machines, stationed at the lowland bases in Zurich, Basel, Bern, Lausanne and St. Gallen, and 11 AgustaWestland Da Vinci located at the mountain bases in Untervaz, Locarno, Erstfeld, Samedan, Wilderswil, Mollis and Zweisimmen. All 13 helicopters are equipped for winch operations and night-time flying.

In Switzerland, the HEMS crew includes a pilot, a paramedic and an emergency physician. The paramedic also serves as winch operator and is therefore not available to treat the patient on-site in case of a winch rescue. In 2013, the AutoPulse ${ }^{\oplus}$ device was added to the standard equipment of the Rega HEMS. Primary missions take place in a large variety of environments and situations, ranging from providing support for ground ambulances in urban areas to autonomous operations in extreme mountainous terrain. We included every operation (primary and secondary mission) during the investigation period.

\section{Protocol}

After every HEMS operation, a variety of information is recorded in the Rega operations database (SAP Software), including whether the MCD was used or not. In a first step, we filtered the database for all operations where the use of the MCD had been reported, and we excluded other operations. For the included operations, we then gathered the physicians' medical operations report forms from the Rega archive. In a second step, we examined these reports and entered key information in a database (Labkey). For the remaining operations, we identified the ones in which the patient was transported to a hospital, and we asked the respective hospitals to provide us with the discharge letters of these patients. In a further step, we analyzed the hospitals' discharge letters and also integrated their key information into our database. The fourth and final step was to analyze the collected data. 


\section{Statistics}

The characteristics of all patients were compared using descriptive statistics.

To further elaborate on factors that are associated with an impaired outcome during AutoPulse ${ }^{\bullet}$ resuscitations, univariate and multivariate logistic regression models were built including the binary variables sex, CPR by layperson, shockable cardiac rhythm as well as the continuous variables age and number of shocks applied. All variables with $p<0.1$ in univariate analysis were included in the multivariate analysis.

The binary variable diagnosis (trauma versus medical diagnosis) was excluded due to a separation problem (i.e. only one survivor in the trauma group). To further assess the impact of the diagnosis variable, a Firth's bias reduced logistic regression model was performed to evade separation [14]. The resulting estimate and standard error for the diagnosis variable remained very large. Hence, the diagnosis variable was excluded for the analysis to avoid bias.

Due to the relevant number of missing data in the variables CPR by layperson $(n=70)$, shockable rhythm $(n=62)$, and survival $(n=70)$, a sensitivity analysis using multiple imputations was performed. All variables of the model were used for imputation in the target variable using the pmm method, generating 10 imputed data sets. Multiple imputation was performed using the mice R-Package [15].

Two-sided $p$-values of $<0.05$ were considered statistically significant. All statistical analyses were performed using R Studio 3.6.0 on macOS 10.15.4.

\section{Results}

In the study period, Rega conducted a total of 22,365 HEMS operations. In 626 operations (3\%) the use of the MCD was reported: 590 of those operations $(2.6 \%)$ could be included (Fig. 1). We had to exclude 36 scene calls in which we could not discern why the MCD was used. One part of this excluded group consisted of calls to the scene where the medical report described a scenario that was highly unlikely to have required the use of a MCD. In these cases, we suspected errors were made in entering information into the database. The other part consisted of operations where the medical report was

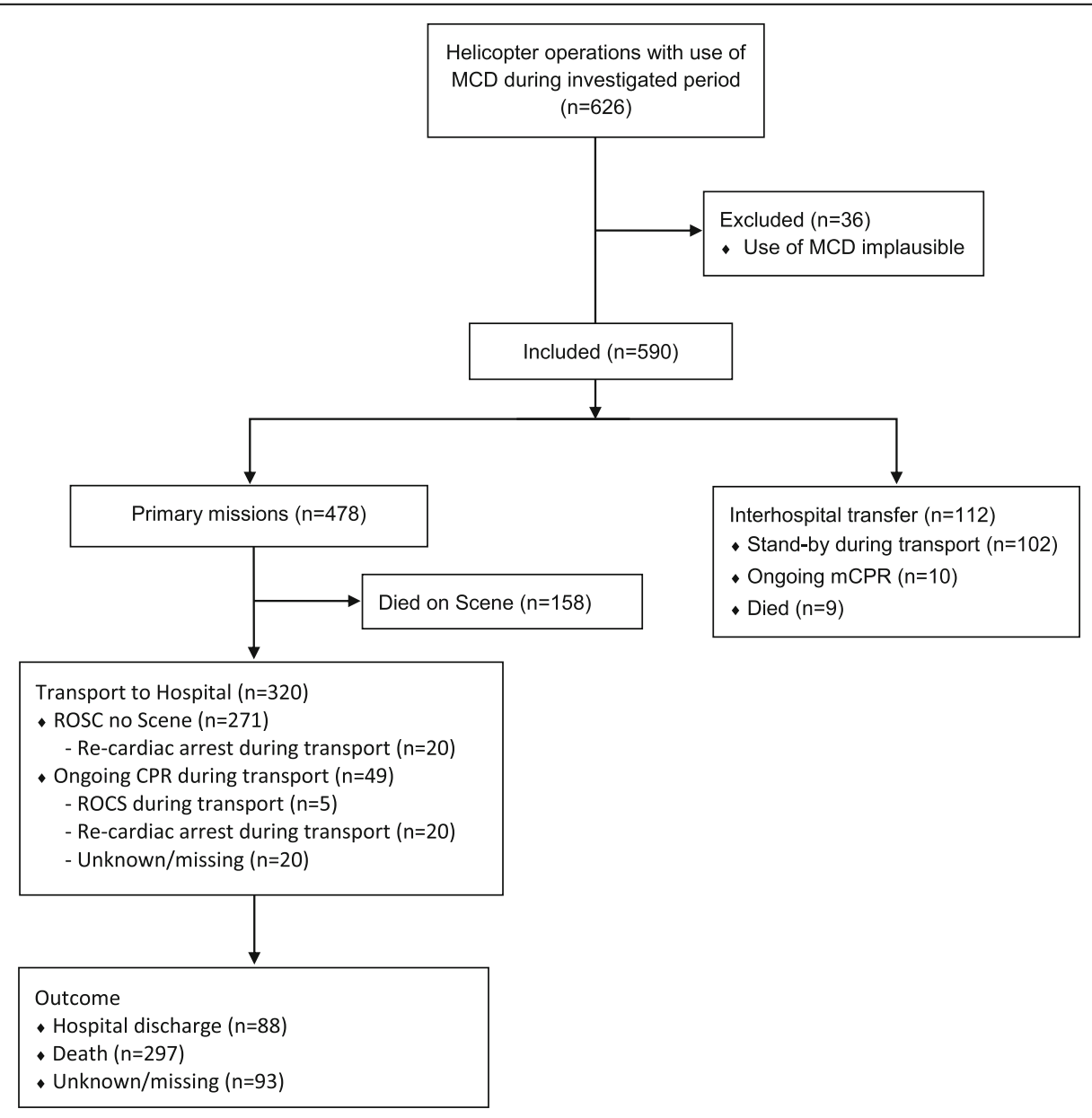

Fig. 1 Flow Diagram of Scene Calls of Primary and Secondary Missions 
missing, destroyed, or unreadable. The included cases could be divided into 478 (81\%) primary and $112(19 \%)$ secondary missions. $74 \%$ of the analysed patients were male, and the mean age was $59 \pm 17$ years. Patient and mission characteristics of primary and secondary missions are described in Tables 1 and 2.

\section{Primary missions}

In 158 cases (33\%), the death of the patient was documented on the scene. In the remaining 320 missions (67\%), the patient was transported to a hospital. The transported patients could be divided into a first group of 49 cases (15\%) where CPR was ongoing when the patient was loaded into the HEMS and a second group of 271 cases $(85 \%)$ where the patient had regained spontaneous circulation before being loaded into the HEMS (Fig. 1). In the first group, ROSC could be achieved during transport in five patients (10\%). However, only one patient (2\%) survival to hospital discharge; 46 patients (94\%) died in the hospital; In two cases (4\%) the endpoint remains unknown because of missing hospital reports. Of the patients in the second group, $20(7 \%)$ experienced a second CA during the flight, two (1\%) could be resuscitated with a single defibrillation attempt, and the remaining 18 (7\%) needed full CPR efforts, including the use of the MCD. Those 18 patients died in

Table 1 Clinical Characteristics of Primary HEMS Missions

\begin{tabular}{|c|c|c|c|c|}
\hline \multirow[t]{2}{*}{ Variable } & \multicolumn{2}{|c|}{ Women, $\boldsymbol{n}=101$ (21\%) } & \multicolumn{2}{|c|}{ Men, $\boldsymbol{n}=377(79 \%)$} \\
\hline & $\begin{array}{l}\text { Trauma } \\
\boldsymbol{n}=17(17 \%)\end{array}$ & $\begin{array}{l}\text { Non-Trauma } \\
\boldsymbol{n}=84(83 \%)\end{array}$ & $\begin{array}{l}\text { Trauma } \\
\boldsymbol{n}=53(14 \%)\end{array}$ & $\begin{array}{l}\text { Non-Trauma } \\
\boldsymbol{n}=324(86 \%)\end{array}$ \\
\hline Age, mean $\pm S D$ & $59 \pm 17$ & $58 \pm 17$ & $53 \pm 17$ & $58 \pm 17$ \\
\hline Autopulse standby & - & - & - & $15(5 \%)$ \\
\hline Asphyxiation/Drowning & $9(53 \%)$ & - & $11(21 \%)$ & - \\
\hline Avalanche/Hypothermia & $2(12 \%)$ & - & $8(15 \%)$ & - \\
\hline Blunt multiple trauma & $6(35 \%)$ & - & $32(60 \%)$ & - \\
\hline Other/Unknown & - & - & $2(4 \%)$ & - \\
\hline Presumed cardiac & - & $27(32 \%)$ & - & 144 (44\%) \\
\hline Respiratory & - & - & - & $4(1 \%)$ \\
\hline Other/Unknown & - & $57(68 \%)$ & - & $176(54 \%)$ \\
\hline Defibrillation & - & $40(48 \%)$ & $5(9 \%)$ & $198(61 \%)$ \\
\hline In flight & - & $1(1 \%)$ & - & $12(4 \%)$ \\
\hline AED & - & $3(4 \%)$ & - & $17(5 \%)$ \\
\hline \multicolumn{5}{|l|}{ Initial cardiac rhythm } \\
\hline VFNT & $1(6 \%)$ & $33(39 \%)$ & $1(2 \%)$ & $168(52 \%)$ \\
\hline PEA/Asystole & $16(94 \%)$ & 33 (39\%) & 49 (92\%) & 109 (34\%) \\
\hline Unknown & - & $18(21 \%)$ & $3(6 \%)$ & 47 (15\%) \\
\hline Lay CPR before EMS arrival & $7(41 \%)$ & $36(43 \%)$ & $25(47 \%)$ & $187(58 \%)$ \\
\hline ROSC & $4(24 \%)$ & $59(70 \%)$ & $20(38 \%)$ & $225(69 \%)$ \\
\hline Intubation & $17(100 \%)$ & $84(100 \%)$ & $53(100 \%)$ & $309(95 \%)$ \\
\hline Transport with ongoing CPR & $3(17 \%)^{*}$ & $8(10 \%)$ & $12(23 \%)^{*}$ & $26(8 \%)$ \\
\hline \multicolumn{5}{|l|}{ Time on scene } \\
\hline$<30 \min$ & $1(6 \%)$ & $13(15 \%)$ & $10(19 \%)$ & $90(28 \%)$ \\
\hline $30-60 \min$ & - & 19 (23\%) & $23(43 \%)$ & $78(24 \%)$ \\
\hline$>60 \min$ & - & $4(5 \%)$ & - & $4(1 \%)$ \\
\hline Unknown & - & - & - & - \\
\hline \multicolumn{5}{|l|}{ Outcome } \\
\hline Hospital discharge & - & $11(13 \%)$ & $1(2 \%)$ & $76(23 \%)$ \\
\hline Death & $17(100 \%)$ & $48(57 \%)$ & $52(98 \%)$ & $180(56 \%)$ \\
\hline Unknown & - & 25 (30\%) & - & $68(21 \%)$ \\
\hline
\end{tabular}

Percentages are calculated within the columns

$S D$ Standard Deviation, AED Automated External Defibrillator, VF Ventricular Fibrillation, VT Ventricular Tachycardia, PEA Pulseless Electrical Activity, CPR Cardiopulmonary Resuscitation, EMS Emergency Medical Services, ROSC Return of Spontaneous Circulation 
Table 2 Clinical Characteristics of Secondary HEMS Missions

\begin{tabular}{llll}
\hline Variable & Overall & Women & Men \\
& $\boldsymbol{N}=112$ & $\boldsymbol{n}=29(26 \%)$ & $\boldsymbol{n}=83(74 \%)$ \\
\hline Age, mean \pm SD & $65 \pm 15$ & $64 \pm 15$ & $65 \pm 14$ \\
Presumed Cardiac & $54(48 \%)$ & $23(79 \%)$ & $31(37 \%)$ \\
Respiratory & $5(5 \%)$ & - & $5(6 \%)$ \\
Other / Unknown & $53(47 \%)$ & $6(21 \%)$ & $47(57 \%)$ \\
Autopulse mode during transport & & \\
$\quad$ Stand-by & $102(91 \%)$ & $25(86 \%)$ & $76(92 \%)$ \\
$\quad$ Active (ongoing CPR) & $9(8 \%)$ & $4(14 \%)$ & $6(7 \%)$ \\
$\quad$ Stand-by/active & $1(1 \%)$ & - & $1(1 \%)$ \\
Outcome & & & $37(45 \%)$ \\
Hospital discharge & $49(44 \%)$ & $12(41 \%)$ & $21(25 \%)$ \\
Death & $31(28 \%)^{*}$ & $10(35 \%)$ & $25(30 \%)$ \\
$\quad$ Unknown & $32(29 \%)$ & $7(24 \%)$ &
\end{tabular}

Percentages are calculated within the columns

CPR Cardiopulmonary Resuscitation

$N=112$, *Autopulse active/ongoing $\mathrm{mCPR}=100 \%$ death

hospital. The remaining 251 patients (93\%) from this group with ROSC on the scene remained haemodynamically stable during the flight.

In two cases $(0.4 \%$ of all primary operations) a malfunction of the MCD was documented. One malfunction was due to rough terrain hindering the correct positioning of the device; the second was an unexplained inability of the device to start chest compressions when cardiac arrest occurred in flight.

The logistic regression models show that presenting with a shockable cardiac arrhythmia is the only factor significantly associated with better survival in resuscitation missions using MCDs (OR 0.176, 95\% confidence interval $[\mathrm{CI}] 0.084$ to $0.372, p<0.001)$. The odds of survival are more than five times greater in patients with a shockable cardiac arrhythmia compared to patients with a non-shockable cardiac rhythm. This finding was robust in the sensitivity analysis using multiple imputation methods for missing data.

Univariate logistic regression models showed that male sex and layperson CPR were associated with better survival as well (Table 3; Fig. 2). However, these effects were no longer statistically significant when adjusted for the type of cardiac arrhythmia (shockable versus nonshockable) in the multivariate analysis (Tables 4 and 5; Fig. 2).

Table 3 HEMS Mission Location

\begin{tabular}{ll}
\hline Location (primary and secondary missions) & $N=590$ \\
\hline Hospital (Interhospital Transfer/secondary missions) & $N=112(19 \%)$ \\
Public place (primary missions) & $N=360(61 \%)$ \\
Mountainous or remote locations (primary missions) & $N=118(20 \%)$ \\
(5 missions with ongoing CPR during winch rescue) & \\
\hline$N=590$ &
\end{tabular}

\section{Secondary missions}

Amongst the secondary operations, 102 (91\%) did not require use of the $\mathrm{MCD}$, which was preinstalled and on standby. Of the rest, five (5\%) patients were loaded into the helicopter with ongoing CPR; ultimately, all of these patients died in the hospital. The remaining five (5\%) patients went into cardiac arrest during the flight and CPR was initiated, with one of them achieving ROSC before landing (Fig. 1).

\section{Discussion}

We found that in successfully resuscitated patients, the risk of cardiac arrest re-occurring during the flight was high during both primary and secondary missions. Therefore, MCDs are beneficial for CPR during airlift to the hospital. Employing MCDs was feasible and safe in the HEMS; standing orders and crew training were carefully implemented, as previously described [16]. There were only two MCD malfunctions (one on the scene, one in flight).

Unnecessary prolonged CPR before terminating efforts is reported in several studies and should be avoided, as it blocks the HEMS crew and therefore wastes EMS and hospital resources $[17,18]$. In our study, the time until ROSC or termination of CPR was usually $<30 \mathrm{~min}$, indicating determined decision-making on the scene in routine cases. However, in patients being airlifted with ongoing CPR, we found that there is a relevant proportion of cases without recommended indication regarding reversible causes of cardiac arrest, suggesting an individual interpretation of the situation. The poor prognosis in this group of patients, with only $3 \%$ survival at discharge, is in accordance with recent studies $[19,20]$. However, precise criteria for patient selection and timing are still lacking. On the other hand, there is an increasing amount of literature showing that carefully selected patients may benefit from early transport with ongoing CPR for further treatment [19-21].

A relevant finding of our study was the fact that in 20 of 271 patients with ROSC (7\%), a second cardiac arrest occurred. A pre-installed MCD was a safe and fast way to initiate chest compressions in flight. However, in many HEMS worldwide, manpower and space in the cabin are severely limited and it is impossible to install a MCD or to perform sufficient and efficient manual chest compressions in flight. Thus our findings support the need for a preinstalled device in this patient group to prevent a relevant delay in CPR in case of re-arrest.

A shockable cardiac arrhythmia was associated with a five times higher chance for survival in the multivariate logistic regression analysis (OR $0.176,95 \%$ CI 0.084 to $0.372, p<0.001)$. These findings are in a line with previous data which demonstrated that survival was best in the group with shockable rhythms in OHCA [22]. These 


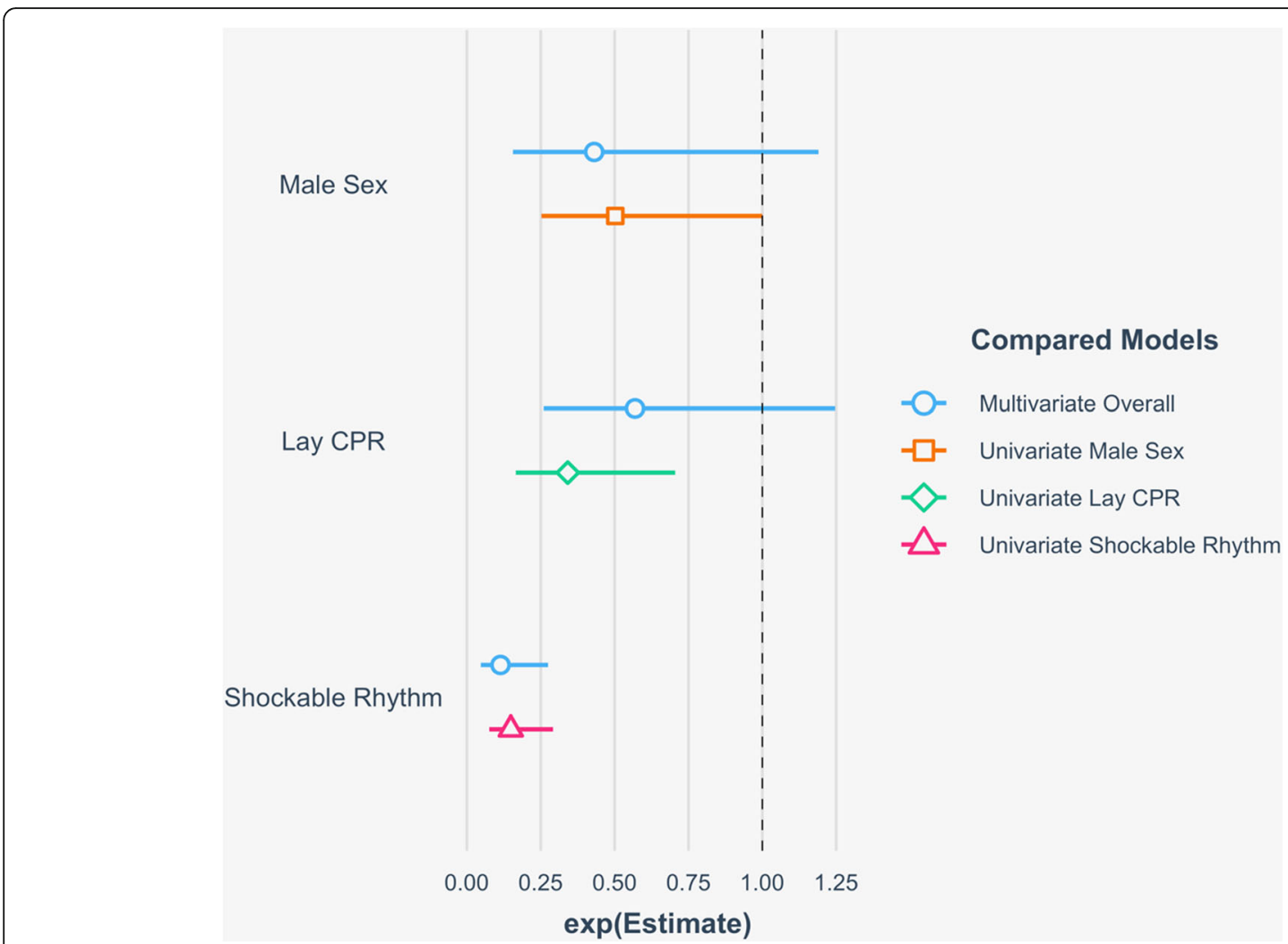

Fig. 2 Univariate compared to Multivariate Logistic Regression Models on Survival in Resuscitations using MCD. Figure based on the CompleteCase Analysis, see Tables 4 and 5. exp. (Estimate): Representing odds ratios, 1.00 indicates no difference in survival. The line around the dot indicates the 95\% Confidence interval of the odds rat

findings could help create further SOPs on the indication, use and duration of MCDs.

Despite the evidence against general use of MCDs, case reports and the recent ERC and AHA guidelines report beneficial effects during prolonged CPR in special circumstances, such as accidental hypothermia, intoxication, or pulmonary embolism [23-25]. However, providing high-quality chest compressions with minimal hands-off time is challenging during evacuation, e.g., in alpine environments and during transport in a helicopter. Accidental hypothermia from a fall in a crevice, submersion in ice water, or burying in an avalanche is more common in HEMS accidents than in accidents treated by ground-based EMS $[25,26]$. Therefore, according to the current CPR recommendations, a robust and feasible way to perform CPR is crucial in this patient group.

Few studies have looked at the feasibility of the different MCDs in mountain HEMS. In general, there are different underlying mechanisms and theories driving MCDs, the cardiac pump theory (LUCAS ${ }^{\mathrm{ma}}$ ) and the thoracic pump theory (Autopulse ${ }^{\circ}$ ) [27]. Requirements for HEMS with winch rescue are different from requirements for ground-based EMS. In a simulation study and additionally in a case series, Pietsch et al. demonstrated the feasibility of using MCDs, even in remote areas and adverse environments requiring winch rescues with

Table 4 Multivariate Logistic Regression Models on Survival in Resuscitations using Autopulse

\begin{tabular}{|c|c|c|c|c|c|c|}
\hline \multirow[t]{2}{*}{ Variable } & \multicolumn{3}{|c|}{ Multivariate Model (Complete Case Analysis) } & \multicolumn{3}{|c|}{ Multivariate Model (Multiple Imputation) } \\
\hline & OR & 95\% C.I. & $p$-value & $\overline{O R}$ & 95\% C.I. & $p$-value \\
\hline Male Sex & 0.456 & 0.167 to 1.248 & 0.126 & 0.672 & 0.338 to 1.336 & 0.258 \\
\hline CPR by Lay & 0.554 & 0.255 to 1.202 & 0.135 & 0.610 & 0.307 to 1.214 & 0.111 \\
\hline Shockable Rhythm & 0.176 & 0.084 to 0.372 & $<0.001$ & 0.092 & 0.073 to 0.286 & $<0.001$ \\
\hline
\end{tabular}

OR Odds ratio, $95 \%$ C.I. $95 \%$ Confidence interval of the odds ratio

Complete-Case Analysis: Included observations $n=315,163$ missing observations

Multiple-Imputation Model: No of Imputations $=10$, No of Iterations $=50$, Method $=$ PMM

Female sex, not receiving lay CPR and non-shockable cardiac rhythm served as the reference groups in both models 
Table 5 Univariate Logistic Regression Models on Survival in Resuscitations using Autopulse

\begin{tabular}{|c|c|c|c|c|c|c|}
\hline \multirow[t]{2}{*}{ Variable } & \multicolumn{3}{|c|}{ Univariate Models (Complete Case Analysis) } & \multicolumn{3}{|c|}{ Univariate Models (Multiple Imputation) } \\
\hline & OR & 95\% C.I. & $p$-value & OR & 95\% C.I. & $p$-value \\
\hline Age & 0.999 & 0.985 to 1.013 & 0.840 & 0.999 & 0.985 to 1.012 & 0.816 \\
\hline Male Sex & 0.502 & 0.252 to 1.000 & 0.050 & 0.325 & 0.284 to 1.012 & 0.057 \\
\hline CPR by Lay & 0.341 & 0.165 to 0.705 & 0.004 & 0.406 & 0.226 to 0.729 & 0.004 \\
\hline Shockable Rhythm & 0.148 & 0.076 to 0.291 & $<0.001$ & 0.128 & 0.065 to 0.255 & $<0.001$ \\
\hline No of Shocks & 0.938 & 0.858 to 1.025 & 0.158 & 0.948 & 0.872 to 1.031 & 0.214 \\
\hline Trauma & 46.08 & 6.279 to 338.2 & $<0.001$ & 30.54 & 4.014 to 232.3 & 0.001 \\
\hline
\end{tabular}

OR Odds ratio, $95 \%$ C.I. $95 \%$ Confidence interval of the odds ratio

Complete-Case Analysis: Included observations $n=315,163$ missing observations

Female sex, not receiving lay CPR and non-shockable cardiac rhythm served as the reference groups in both models

Trauma variable: Due to the low number of survivors in patients with a trauma diagnosis $(n=1)$ the regression estimates are biased (separation); estimates are presented for completeness purpose only

Multiple-Imputation Model: No of Imputations $=10$, No of Iterations $=50$, Method $=$ PMM

ongoing chest compression [4, 28]. In our study, one of the 69 trauma patients with known data survived to hospital discharge. This may indicate that the trauma patient sample was too small, or that the wrong patients were transported to the hospital.

All of the existing MCD studies, and even the recent Cochrane review, excluded studies explicitly including patients with cardiac arrest caused by trauma, drowning, hypothermia and toxic substances. These conditions are mostly excluded from cardiac arrest intervention studies because they have a different underlying pathophysiology, therapy and thus prognosis [29]. To our knowledge there is no study reporting data on outcomes of traumatic CA and MCDs so far. Thus, this study would be the first reporting data in this field of non-cardiac-caused CAs and MCDs. In contrast, hospital discharge rates ranged between $13 \%$ in women and $23 \%$ in men suffering nontraumatic cardiac arrest, indicating that employing MCDs resulted in very good survival rates in this group. Our clear findings against the use of MCDs in traumatic CA could potentially influence resuscitation guidelines in the future.

MCDs offer additional benefits for the HEMS crew. During a simulated helicopter transport, Rehatschek et al. showed that MCDs improved the efficacy of chest compressions, reduced physical stress, and led to enhanced cognitive performance of the EMS crew as compared to manual CPR [30]. This may enable better monitoring of clinical developments, and leaves hands free for additional interventions. However, a theoretical basis and evidence regarding those positive effects on human error, as well as data on non-technical skills, are still limited.

An obvious reason to implement MCDs in HEMS is to ensure conformity with flight transport regulations, such as the requirement for the entire crew to have latched seatbelts during ongoing chest compressions in flight. Further, with a growing number of contagious diseases (e.g., COVID-19), MCDs can enhance safety while still allowing provision of high-quality CPR with maximal distance to the patient on the scene and during transport.

The strength of our study is the large patient cohort we examined. The HEMS missions in our study are very diverse, consisting of a mixture of urban, rural, mountainous, and alpine areas, thus the results may be extrapolated to other EMS settings. Due to the retrospective study design we could investigate application of MCDs in daily practice of HEMS without the artificial limitations of a prospective trial protocol. In addition, the fact that the involved HEMS were physician-staffed meant that the course of CPR efforts in our study was not heavily influenced by legal considerations, since physicians could pronounce a patient dead on the scene. In contrast, paramedic-staffed services - depending on local legislation and protocols - might require extensive CPR efforts before termination, thus altering the role of MCDs.

The retrospective observational nature of our study has several limitations as well. First, there was no systematically gathered information regarding MCDs from the HEMS missions except for the question of whether the device was used or not. We extracted all relevant information from the medical HEMS reports, but quality varied greatly. Second, there may have been missions where a MCD was used but not documented in the HEMS database. Since we were unable to check all missions manually, we could not include them, and therefore we might have underestimated the true proportion of HEMS missions involving MCDs, a potential source of selection bias. Third, a multivariable logistic regression was performed adjusting the analysis for confounding factors, however, residual confounding due to unmeasured factors is possible. And last, we could not compare our findings with a control group since identifying a set of comparable HEMS missions in the years preceding the implementation of MCDs in our organization was not possible. 


\section{Conclusion}

\section{Equipping HEMS with MCDs may be beneficial to treat patients with non-traumatic cardiac arrests.}

\section{Abbreviations}

AED: Automated external defibrillator; AHA: American Heart Association CA: Cardiac arrest; CPR: Cardiopulmonary resuscitation; ECMO: Extracorporeal membrane oxygenation; ECPR: Extracorporeal cardiopulmonary resuscitation; ERC: European Resuscitation Council; HEMS: Helicopter emergency medical service; ILCOR: International Liaison Committee on Resuscitation; KEK: Cantonal Ethics Committee; LUCAS: Lund University Cardiac Assist System; MCD: Mechanical chest compression device; NACA: National Advisory Committee for Aeronautics; OHCA: Out-of-hospital cardiac arrest

\section{Acknowledgements}

We wish to thank all involved paramedics, pilots and physicians for their contributions. We would like to thank Jeannie Wurz, Medical Editor, Bern, Switzerland, for editorial assistance.

\section{Authors' contributions}

UP and RA conceived the study. UP and DR obtained the data. UP, LT, DR, LM, WW and JK conducted the analysis. UP, SR and WW drafted the manuscript and all authors contributed to its critical review. UP assumes overall responsibility for the paper. All authors read and agreed to the final version of the manuscript.

\section{Funding}

None.

\section{Availability of data and materials}

Not applicable.

\section{Ethics approval and consent to participate}

The institutional review board of the Bern cantonal ethics committee (KEK) reviewed the study design and granted permission for use of patient data without individual consent, according to the federal act on research involving human beings, and the ordinance on human research with the exception of clinical trials. The permission covered the use of patient data collected during the HEMS operation and related hospital stays (KEK Bern, September 13, 2016, reference number 2016-01473).

\section{Consent for publication}

Not applicable.

\section{Competing interests}

The authors declare that they have no competing interest.

\section{Author details}

${ }^{1}$ Department of Anaesthesiology and Intensive Care Medicine, Cantonal Hospital St. Gallen, Rorschacher Strasse 95, 9007 St. Gallen, Switzerland. ${ }^{2}$ Air Zermatt, Emergency Medical Service, Zermatt, Switzerland. ${ }^{3}$ Department of Anaesthesiology and Pain Medicine, Inselspital, Bern University Hospital, University of Bern, Bern, Switzerland. ${ }^{4}$ Department of Anaesthesiology and Intensive Care Medicine, Friedrichshafen Regional Hospital, Röntgenstraße 2, 88048 Friedrichshafen, Germany. ${ }^{5}$ Swiss Air-Ambulance, Rega

(Rettungsflugwacht / Guarde Aérienne), Swiss Air-Rescue, Zurich, Switzerland. ${ }^{6}$ Institute of Mountain Emergency Medicine, Eurac Research, Bozen, Italy. ${ }^{7}$ Department of Anaesthesiology and Intensive Care Medicine, F. Tappeiner Hospital, Merano, Italy. ${ }^{8}$ Department of Vascular Surgery, St. Gallen Cantonal Hospital, St. Gallen, Switzerland.

Received: 14 May 2020 Accepted: 24 June 2020

Published online: 25 July 2020

\section{References}

1. Vadeboncoeur T, Stolz U, Panchal A, Silver A, Venuti M, Tobin J, et al. Chest compression depth and survival in out-of-hospital cardiac arrest. Resuscitation. 2014;85(2):182-8.

2. Idris AH, Guffey D, Pepe PE, Brown SP, Brooks SC, Callaway CW, et al. Chest compression rates and survival following out-of-hospital cardiac arrest. Crit Care Med. 2015;43(4):840-8.
3. Perkins GD, Handley AJ, Koster RW, Castren M, Smyth MA, Olasveengen T, et al. European resuscitation council guidelines for resuscitation 2015: section 2. Adult basic life support and automated external defibrillation. Resuscitation. 2015:95:81-99.

4. Pietsch U, Lischke V, Pietsch C, Kopp KH. Mechanical chest compressions in an avalanche victim with cardiac arrest: an option for extreme mountain rescue operations. Wilderness Environ Med. 2014;25(2):190-3.

5. Havel C, Schreiber W, Riedmuller E, Haugk M, Richling N, Trimmel H, et al. Quality of closed chest compression in ambulance vehicles, flying helicopters and at the scene. Resuscitation. 2007;73(2):264-70.

6. Omori K, Sato S, Sumi Y, Inoue Y, Okamoto K, Uzura M, et al. The analysis of efficacy for AutoPulse\&\#x2122; system in flying helicopter. Resuscitation. 2013:84(8):1045-50.

7. Wroe PC, Clattenburg EJ, Gardner K, Gelber J, Schultz C, Singh A, et al. Emergency department use of a mechanical chest compression device frequently causes unanticipated interruptions in cardiopulmonary resuscitation. Resuscitation. 2018;133:e3-4.

8. Schmidbauer S, Herlitz J, Karlsson T, Axelsson C, Friberg H. Use of automated chest compression devices after out-of-hospital cardiac arrest in Sweden. Resuscitation. 2017;120:95-102.

9. Rubertsson S. Update on mechanical cardiopulmonary resuscitation devices. Curr Opin Crit Care. 2016;22(3):225-9.

10. Rubertsson S, Lindgren E, Smekal D, Ostlund O, Silfverstolpe J, Lichtveld RA, et al. Mechanical chest compressions and simultaneous defibrillation vs conventional cardiopulmonary resuscitation in out-of-hospital cardiac arrest: the LINC randomized trial. Jama. 2014;311(1):53-61.

11. Perkins GD, Lall R, Quinn T, Deakin CD, Cooke MW, Horton J, et al. Mechanical versus manual chest compression for out-of-hospital cardiac arrest (PARAMEDIC): a pragmatic, cluster randomised controlled trial. Lancet. 2015;385(9972):947-55.

12. Lerner EB, Persse D, Souders CM, Sterz F, Malzer R, Lozano M Jr, et al. Design of the Circulation Improving Resuscitation Care (CIRC) trial: a new state of the art design for out-of-hospital cardiac arrest research. Resuscitation. 2011:82(3):294-9.

13. Yates EJ, Schmidbauer S, Smyth AM, Ward M, Dorrian S, Siriwardena AN, et al. Out-of-hospital cardiac arrest termination of resuscitation with ongoing CPR: an observational study. Resuscitation. 2018;130:21-7.

14. Heinze G, Ploner M. A SAS-macro, S-PLUS library and R package to perform logistic regression without convergence problems. Technical report 2/2004; 2004.

15. Buuren S, Groothuis-Oudshoorn C. MICE: multivariate imputation by chained equations in R. J Stat Softw. 2011:45:1.

16. Rauch S, Strapazzon G, Brodmann M, Fop E, Masoner C, Rauch L, et al. Implementation of a mechanical CPR device in a physician staffed HEMS - a prospective observational study. Scand J Trauma Resusc Emerg Med. 2018; 26(1):36.

17. Hallstrom A, Rea TD, Sayre MR, Christenson J, Anton AR, Mosesso VN Jr, et al. Manual chest compression vs use of an automated chest compression device during resuscitation following out-of-hospital cardiac arrest: a randomized trial. Jama. 2006;295(22):2620-8.

18. Bonnes JL, Brouwer MA, Navarese EP, Verhaert DV, Verheugt FW, Smeets JL, et al. Manual cardiopulmonary resuscitation versus CPR including a mechanical chest compression device in out-of-hospital cardiac arrest: a comprehensive meta-analysis from randomized and observational studies. Ann Emerg Med. 2016;67(3):349-60.e3.

19. Adler C, Paul C, Michels G, Pfister R, Sabashnikov A, Hinkelbein J, et al. One year experience with fast track algorithm in patients with refractory out-ofhospital cardiac arrest. Resuscitation. 2019;144:157-65.

20. Adler C, Paul C, Hinkelbein J, Michels G, Pfister R, Krings A, et al. Welcher patient profitiert von einem transport unter laufender kardiopulmonaler reanimation? Anaesthesist. 2018;67(5):343-50.

21. Spangenberg T, Meincke F, Brooks $S$, Frerker $C$, Kreidel F, Thielsen T, et al. "Shock and go?" extracorporeal cardio-pulmonary resuscitation in the golden-hour of ROSC. Catheter Cardiovasc Interv. 2016;88(5):691-6.

22. Vukmir RB. Initial cardiac rhythm correlated to emergency department survival. Clin Med Cardiol. 2009:3:9-14.

23. McCormack J, Percival D. HEMS advanced trauma team retrieval of a patient with accidental hypothermic cardiac arrest for ECMO therapy. Resuscitation. 2016;105:e23.

24. Lyon R, Nelson M. Helicopter emergency medical services (HEMS) response to out-of-hospital cardiac arrest. Resuscitation. 2012;83:e96. 
25. Métrailler-Mermoud J, Hugli O, Carron P-N, Kottmann A, Frochaux V, ZenRuffinen $\mathrm{G}$, et al. Avalanche victims in cardiac arrest are unlikely to survive despite adherence to medical guidelines. Resuscitation. 2019;141:35-43.

26. Holmström P, Boyd J, Sorsa M, Kuisma M. A case of hypothermic cardiac arrest treated with an external chest compression device (LUCAS) during transport to re-warming. Resuscitation. 2005;67(1):139-41.

27. Haas T, Voelckel WG, Wenzel V, Antretter H, Dessl A, Lindner KH. Revisiting the cardiac versus thoracic pump mechanism during cardiopulmonary resuscitation. Resuscitation. 2003;58(1):113-6.

28. Pietsch U, Lischke V, Pietsch C. Benefit of mechanical chest compression devices in mountain HEMS: lessons learned from 1 year of experience and evaluation. Air Med J. 2014;33(6):299-301.

29. Wang PL, Brooks SC. Mechanical versus manual chest compressions for cardiac arrest. Cochrane Database Syst Rev. 2018;8(8):CD007260.

30. Rehatschek G, Muench M, Schenk I, Dittrich W, Schewe JC, Dirk C, et al. Mechanical LUCAS resuscitation is effective, reduces physical workload and improves mental performance of helicopter teams. Minerva Anestesiol. 2016;82(4):429-37.

\section{Publisher's Note}

Springer Nature remains neutral with regard to jurisdictional claims in published maps and institutional affiliations.

Ready to submit your research? Choose BMC and benefit from:

- fast, convenient online submission

- thorough peer review by experienced researchers in your field

- rapid publication on acceptance

- support for research data, including large and complex data types

- gold Open Access which fosters wider collaboration and increased citations

- maximum visibility for your research: over $100 \mathrm{M}$ website views per year

At BMC, research is always in progress.

Learn more biomedcentral.com/submissions 